\title{
Keeping secrets: the economics of access deterrence
}

\author{
Emeric Henry Francisco Ruiz-Aliseda \\ ONLINE APPENDIX
}


This appendix extends our results to the case in which protection not only requires that an insider has paid $c_{p}$ upon entry, but also that she has never stopped paying the per-period fixed cost $f>0$ at some point in time in the past. Therefore, the sole modification with respect to the baseline model is that, in any period, if all insiders have incurred the protection cost $c_{p}$ and continuously paid $f$, the outsiders are assumed to be able to instantaneously enter by paying $c_{e}>0$; however, if one of the insiders did not pay $c_{p}$ upon entry or stopped paying $f$, then entry would become costless for all outsiders. Insiders and outsiders take actions simultaneously at each point in time, with all past actions being observable. In each period, an outsider must therefore choose whether to take action $p$, action $u$ or action $w$. In turn, an insider must choose whether to pay the protection cost $f$ (an action denoted by $\widehat{p}$ ) or not to incur it (an action denoted by $\widehat{u}$ ). Throughout, we will let $F \equiv f / r$.

We first study the case in which $\Pi_{n}>c_{e}$, mentioning at the end of the appendix how to deal with the cases in which such condition is violated. Before starting the equilibrium analysis, let us clarify several points. First, we note that in any subgame in which at least one of the insiders did not pay for protection in the past (either did not pay $c_{p}$ upon entry or stopped paying $f$ at some point after entry), all outsiders immediately enter for free. Thus in the following discussion, we exclusively focus on subgames in which all insiders have paid for protection at each point in time until the beginning of the subgame.

Second, we point out that, in any subgame with $k \leq n-2$ outsiders, there always exist equilibria such that no insider pays $f$ and all the outsiders then immediately enter at zero cost. Such an equilibrium is a normal outcome in coordination games. As we explained in the paper's main text, we shall refer to such continuation equilibria in subgames with $k \leq n-2$ outsiders as "no-barrier continuation equilibria" (with the implicit understanding that the continuation subgame to which they refer is the one with $k$ outsiders). For certain range of parameters, there will be other equilibria in which all the insiders will find it optimal to pay $f$ in the subgame under consideration. We will call those "barrier continuation equilibria." 
Third, note that a key consideration when we study subgames with $k$ outsiders left is what equilibria will be played in subgames following entry of one of the outsiders, i.e., subgames with $k^{\prime}<k$ outsiders. In particular, if it is expected that no-barrier equilibria will be played in all following subgames, all players know that after the next entry, all outsiders will enter for free. This consideration therefore leads to a multiplicity of equilibria. To reduce the number of cases under consideration, we shall restrict attention to the following class of equilibria: if, in a subgame with $k$ outsiders, a no-barrier (continuation) equilibrium is played, then no-barrier equilibria are played in all subgames with less than $k$ outsiders. ${ }^{1}$ Technically, this preserves some monotonicity in the continuation payoffs of insiders that are useful to determine the payoff in the preemption phase: in that phase, given that the number of outsiders who enter is random, all continuation subgames are possible outcomes. Intuitively, these equilibria also appear very reasonable: if it is not possible to coordinate on paying $f$ for a subgame with $k$ outsiders and $n-k$ insiders, it should be even more difficult when there are more insiders.

Finally, we note that we will not directly use a proof by induction as we did in the paper's appendix in order to illustrate how we compute the continuoustime limit of the game given the randomizing behavior by game players.

\section{Solving the subgames with less than three outsiders}

\section{One outsider left to enter}

We work backwards in the state space, so we begin our analysis by considering those subgames in which just one outsider is left to enter, that is, $k=1$. Since $\Pi_{n}>c_{e}$, it is a dominant strategy for the unique outsider to enter immediately, so no insider ever pays for protection. The continuation payoff of an insider in such a subgame is then $I_{1}=\Pi_{n}$. The continuation payoff of the outsider is $O_{1}=\Pi_{n}$.

\section{Two outsiders left to enter}

\footnotetext{
${ }^{1}$ It is important to take into account that if a barrier equilibrium exists in a subgame with $k$ outsiders, then there exists a barrier equilibrium in any subgame with fewer outsiders.
} 
We now consider the subgames with only two outsiders left (i.e., $k=2$ ). As pointed out above, there always exist "no-barrier equilibria" in which insiders never pay $f$ in this subgame and both outsiders enter immediately as soon as the subgame starts. If $F$ is large $\left(F>\Pi_{n-2}-\Pi_{n}\right)$, this is the unique equilibrium outcome of this subgame. When $F<\Pi_{n-2}-\Pi_{n}$, we proceed to show that there exists another equilibrium in which insiders continuously pay $f$ as long as no entry takes place. This behavior by insiders, aimed at keeping the entry cost at $c_{e}$, creates the same dilemma for the two remaining outsiders as the one that we studied in the paper's main text, so they will mix between entering without paying the protection cost and waiting. The following lemma formalizes the possible equilibrium outcomes in these subgames as we work in continuous time.

Lemma A In subgames with two outsiders:

1. There always exists a no-barrier continuation equilibrium such that insiders do not pay $f$ and outsiders choose action $u$; the continuation payoff of an outsider is $\mathrm{O}_{2}=\Pi_{n}$, whereas the continuation payoff of an insider is $I_{2}=\Pi_{n}$. If $F>\Pi_{n-2}-\Pi_{n}$, this is the unique (symmetric) equilibrium.

2. If $F<\Pi_{n-2}-\Pi_{n}$, there also exists a barrier continuation equilibrium such that insiders pay $f$ as long as the subgame is not over and outsiders mix between actions $u$ and $w$ at each point in time. In the continuoustime limit of the game, the entry time of an outsider is drawn from an exponential distribution with parameter $\lambda_{2}$, where $\lambda_{2} \equiv r\left(\Pi_{n}-c_{e}\right) / c_{e}$. The continuation payoff of an outsider is $O_{2}=\Pi_{n}-c_{e}$, whereas the continuation payoff of insiders is $I_{2}=\mu_{2}\left(\Pi_{n-2}-F\right)+\left(1-\mu_{2}\right) \Pi_{n}$, where $\mu_{2} \equiv r /\left(r+2 \lambda_{2}\right)$.

Proof. This lemma is a particular case (for $k=2$ ) of a general situation involving $2 \leq k \leq n-1$ outsiders and $n-k$ insiders under the restriction that no outsider ever takes action $p$. We do the proof for general $k$ as it will be used to show later results. For the case of $k=2$ (Lemma A), action $p$ is 
dominated for outsiders. We characterize the equilibrium of this game in a sequence of steps. First, we rule out mixing by the $n-k$ insiders. Next, we characterize the probability with which each of the $k$ outsiders takes action $u$ at each point in time (as long as no outsider has yet entered). Finally, we characterize continuation payoffs to the $k$ outsiders and the $n-k$ insiders.

We proceed by proving first the following claim: if in the subgame with $k$ outsiders, these players mix between actions $u$ and $w$, then the existing $n-k$ insiders cannot be mixing between actions $\widehat{u}$ and $\widehat{p}$ in a symmetric equilibrium. Given that outsiders each choose $u$ with (behavioral) probability $\rho_{u, k} \in(0,1)$ at each period in which the subgame is not over, suppose to the contrary that the existing $n-k$ insiders pay $f$ with (per-period) probability $\varphi_{k} \in(0,1)$.

To ease notation, normalize to zero the date at which the subgame under consideration starts, and let $V_{\widehat{p}, k}(\tau)$ denote an insider's expected payoff from paying $f$ until time $\tau>0$ given that the other $n-k-1$ insiders pay it with probability $\varphi_{k}$ and the $k$ outsiders play $u$ with probability $\rho_{u, k}$. If there are $\tau / \Delta$ periods of length $\Delta>0$ each until $\tau$ is reached, it then follows that

$$
\begin{aligned}
V_{\widehat{p}, k}(\tau)= & \sum_{s=0}^{\frac{\tau}{\Delta}-1}\left(\varphi_{k}^{n-k-1}\left(1-\rho_{u, k}\right)^{k}\right)^{s}\left(1-\varphi_{k}^{n-k-1}\left(1-\rho_{u, k}\right)^{k}\right)\left(\sum_{l=0}^{s-1}\left(\pi_{n-k}-f\right) \Delta \delta^{l}+\sum_{l=s}^{\infty} \pi_{n} \Delta \delta^{l}\right) \\
& +\left(\varphi_{k}^{n-k-1}\left(1-\rho_{u, k}\right)^{k}\right)^{\frac{\tau}{\Delta}}\left(\sum_{l=0}^{\frac{\tau}{\Delta}-1}\left(\pi_{n-k}-f\right) \Delta \delta^{l}+\sum_{l=\frac{\tau}{\Delta}}^{\infty} \pi_{n} \Delta \delta^{l}\right),
\end{aligned}
$$

where we have used the fact that $\int_{0}^{\Delta} \pi_{n-j} e^{-r s} d s \approx \pi_{n-j} \Delta$ for small $\Delta>0 .{ }^{2}$ Note that some straightforward manipulations yield that the variation in the insider's payoff if she pays $f$ one more period (conditional on the subgame not being over) equals

$V_{\widehat{p}, k}(\tau+\Delta)-V_{\widehat{p}, k}(\tau)=\left(\varphi_{k}^{n-k-1}\left(1-\rho_{u, k}\right)^{k}\right)^{\frac{\tau}{\Delta}} \varphi_{k}^{n-k-1}\left(1-\rho_{u, k}\right)^{k}\left(\pi_{n-k}-\pi_{n}-f\right) \Delta \delta^{\frac{\tau}{\Delta}}$.

Because $\operatorname{sign}\left(V_{\widehat{p}, k}(\tau+\Delta)-V_{\widehat{p}, k}(\tau)\right)=\operatorname{sign}\left(\pi_{n-k}-\pi_{n}-f\right)$, we find that when

\footnotetext{
${ }^{2}$ Recall that the end of the current subgame results in immediate entry by the remaining outsiders, thus effectively ending the overall game.
} 
all other insiders pay $f$ at each period with some probability $\varphi_{k} \in(0,1)$ (and outsiders enter with probability $\left.\rho_{u, k} \in(0,1)\right)$ that an insider would find it optimally to unilaterally deviate. In particular, she would prefer paying $f$ with probability one until entry occurred if $f<\pi_{n-k}-\pi_{n}$ (equivalently, if $\left.F<\Pi_{n-k}-\Pi_{n}\right)$, and she would prefer not to ever put some positive weight in paying $f$ at any point in time otherwise.

The consequence is that equilibria must involve either all insiders continuously paying $f$ until entry by some outsider occurs or neither of them ever paying $f$. It is clear that an equilibrium in which no insider pays $f$ always exists and it is such that $I_{k}=\Pi_{n}$ and $O_{k}=\Pi_{n}$. In addition, using the same proof above for $\varphi_{k}=1$ shows that the existing $n-k$ insiders have an incentive to choose $\varphi_{k}=1$ if $F<\Pi_{n-k}-\Pi_{n}$ regardless of the value taken by $\rho_{u, k}$ (if $F \geq \Pi_{n-k}-\Pi_{n}$, only $\varphi_{k}=0$ is possible). That is, when $F<\Pi_{n-k}-\Pi_{n}$, there exists another equilibrium in which insiders pay $f$ as long as entry does not occur and outsiders engage in a waiting game to determine who pays the entry $\operatorname{cost} c_{e}$. We proceed to characterize behavior by outsiders in such an equilibrium exhibiting delay.

To find out the value of $\rho_{u, k}$, let $\varphi_{k}=1$ and let $V_{u, k}(\tau)$ denote the payoff of an outsider to choosing $u$ at time $\tau>0$ given that each of the other $k-1$ outsiders choose such an action with probability $\rho_{u, k}$ at each period of length $\Delta>0$ and wait with complementary probability. It readily follows that

$V_{u, k}(\tau)=\sum_{s=0}^{\frac{\tau}{\Delta}-1}\left[\left(1-\rho_{u, k}\right)^{k-1}\right]^{s}\left[1-\left(1-\rho_{u, k}\right)^{k-1}\right]\left(\sum_{l=s}^{\infty} \pi_{n} \Delta \delta^{l}\right)+\left[\left(1-\rho_{u, k}\right)^{k-1}\right]^{\frac{\tau}{\Delta}}\left(\sum_{l=\frac{\tau}{\Delta}}^{\infty} \pi_{n} \Delta \delta^{l}-c_{e} \delta^{\frac{\tau}{\Delta}}\right)$,

so we have

$$
V_{u, k}(\tau+\Delta)-V_{u, k}(\tau)=\delta^{\frac{\tau}{\Delta}}\left[\left(1-\rho_{u, k}\right)^{k}\right]^{\frac{\tau}{\Delta}}\left[c_{e}-\left(1-\rho_{u, k}\right)^{k-1}\left(\pi_{n} \Delta+\delta c_{e}\right)\right] .
$$

In order for an outsider to be indifferent among choosing action $u$ at all points in time, we must have that $V_{u, k}(\tau+\Delta)=V_{u, k}(\tau)$ for all $\tau$. Since $\delta \approx 1-r \Delta$ 
for small enough $\Delta>0$, it follows that

$$
\rho_{u, k}=1-\left(1+\lambda_{2} \Delta\right)^{-1 /(k-1)},
$$

where $\lambda_{2} \equiv r\left(\Pi_{n}-c_{e}\right) / c_{e}$.

Given some fixed time $\tau>0$, and $m(\Delta)=\tau / \Delta$ periods of play until then, the probability that an outsider does not choose action $u$ at some time earlier than $\tau$ converges to

$$
1-\lim _{\Delta \rightarrow 0}\left(1-\rho_{u, k}\right)^{m(\Delta)}=1-\lim _{\Delta \rightarrow 0}\left(1+\lambda_{2} \Delta\right)^{-\frac{\tau}{(k-1) \Delta}}=1-\lim _{\Delta \rightarrow 0}\left[\left(1+\lambda_{2} \Delta\right)^{\frac{1}{\lambda_{2} \Delta}}\right]^{-\frac{\lambda_{2} \tau}{k-1}}=1-e^{-\frac{\lambda_{2} \tau}{k-1}} .
$$

Using the fact $\lambda_{2} /(k-1)=r\left(\Pi_{n}-c_{e}\right) /\left((k-1) c_{e}\right)$, it then follows that an outsider chooses the first time at which to select action $u$ according to an exponential distribution with parameter $\lambda_{k} \equiv r\left(\Pi_{n}-c_{e}\right) /\left((k-1) c_{e}\right)$.

In a mixed-strategy Nash equilibrium, an outsider should be indifferent among all possible times at which to choose action $u$, so the fact that $V_{u, k}(\Delta)=$ $\Pi_{n}-c_{e}$ implies that the expected gain of an outsider is $O_{k}=\Pi_{n}-c_{e}$. In addition, using the fact that each outsider chooses her entry time according to an exponential distribution with parameter $\lambda_{k}$, the continuation payoff of an insider is

$$
I_{k}=\int_{0}^{\infty}\left(\int_{0}^{\widehat{\tau}}\left(\pi_{n-k}-f\right) e^{-r s} d s+\int_{\widehat{\tau}}^{\infty} \pi_{n} e^{-r s} d s\right) k \lambda_{k} e^{-k \lambda_{k} \widehat{\tau}} d \widehat{\tau} .
$$

Integrating and letting $\mu_{k} \equiv r /\left(r+k \lambda_{k}\right)$ then yields that the continuation payoff of an insider equals

$$
I_{k}=\mu_{k}\left(\Pi_{n-k}-F\right)+\left(1-\mu_{k}\right) \Pi_{n} .
$$

\section{Three outsiders left to enter}

As before, if $F>\Pi_{n-3}-\Pi_{n}$, there can be no equilibria in these subgames other than no-barrier ones. If $F<\Pi_{n-3}-\Pi_{n}$, no-barrier equilibria still exist, 
but there may also exist barrier equilibria that we proceed to characterize. In particular, we show below that sustaining barrier equilibria in the subgame with three outsiders can be based on the expectation that it will be followed by either a no-barrier continuation equilibrium play or a barrier continuation equilibrium in the subgame in which two outsiders are left. Suppose first that a no-barrier equilibrium outcome is expected to occur in the subgame with two outsiders. It then follows that action $p$ (enter and pay the fixed protection cost) is dominated in the current subgame with three outsiders since no one will pay $f$ later on. This creates, as in the previous lemma, the conditions for a waiting game in which the three outsiders try to free ride on each other so as to save on the entry $\operatorname{cost} c_{e}$. We show in the next lemma that the individual entry time then follows an exponential distribution with parameter $\lambda_{3} \equiv r\left(\Pi_{n}-c_{e}\right) /\left(2 c_{e}\right)$.

Suppose now that a barrier equilibrium outcome is expected to occur in the subgame with two outsiders. We examine whether barrier equilibria in the current subgame can be sustained by such an expectation, leaving insiders' incentives to pay $f$ aside for the moment and just focusing on outsiders' incentives. By the previous lemma, an outsider that enters first by paying $c_{p}$ expects a continuation payoff of $I_{2}=\mu_{2}\left(\Pi_{n-2}-F\right)+\left(1-\mu_{2}\right) \Pi_{n}$, so it follows that playing action $p$ is (weakly) dominated by $u$ if $I_{2}-c_{p}-c_{e} \leq O_{2}-c_{e}$. Given that $O_{2}=\Pi_{n}-c_{e}$, the condition can be equivalently expressed as $c_{p} \geq c_{2, F}^{*} \equiv \mu_{2}\left(\Pi_{n-2}-\Pi_{n}-F\right)$. As we just saw above, in this case, the three outsiders will play a waiting game, hoping that another outsider will enter before them. In order for insiders to have an incentive to pay $f$, it is necessary that $F$ not be too large $\left(F<\Pi_{n-3}-\Pi_{n}\right)$.

So the three outsiders left to enter in the subgames under consideration can find themselves in a waiting game for different reasons. As explained above, this can occur because coordination on paying $f$ is expected to fail in the next subgame (no-barrier equilibria will be played) or because of the expectation that an outsider will find it too costly to pay for protection when entering even if she foresees that a barrier equilibrium will be played if she enters (barrier equilibria will be played with $c_{p} \geq c_{2, F}^{*}$ and $F<\Pi_{n-3}-\Pi_{n}$ ). 
In both cases, paying $c_{p}$ is dominated in the current subgame, and any entry will thus be followed by entry of all the remaining outsiders, so the outsiders wait to enter in the hope of saving $c_{e}$. In the lemma below, both of these cases are summarized in part 2a.

If it instead holds that $c_{p}<c_{2, F}^{*}$ (which necessarily requires that $F<$ $\Pi_{n-2}-\Pi_{n}$ ), preemptively entering and paying $c_{p}$ becomes very attractive for an outsider if the other two do not enter and a barrier equilibrium is foreseen in the subgame with two outsiders left. There is however a risk of coordination failure were all outsiders simultaneously to enter and pay $c_{p}$. This creates the conditions for a preemption game described in the lemma below, part $2 \mathrm{~b}$. As the time between two consecutive periods shrinks, outsiders essentially mix between $p$ and $w$, that is, between entering with protection and waiting. Entry occurs instantaneously with probability one, and the number of entrants follows a binomial distribution (so that there is positive probability of simultaneous entry by several outsiders). Because insiders pay $f$ over a negligible amount of time, in the limit there is no constraint on how small $F$ should be (other than the condition initially imposed that $F$ be smaller than $\Pi_{n-2}-\Pi_{n}$ ).

Lemma B In subgames with three outsiders:

1. There always exists a no-barrier continuation equilibrium such that insiders never pay $f$ and outsiders choose action u immediately. If $F>$ $\Pi_{n-3}-\Pi_{n}$, this is the unique (symmetric) equilibrium.

2. If $F<\Pi_{n-3}-\Pi_{n}$ :

(a) There also exist barrier continuation equilibria such that insiders pay $f$ while the subgame is not over and outsiders mix between actions $u$ and $w$ at each point in time. In the continuous-time limit of the game, the entry time of an outsider is drawn from an exponential distribution with parameter $\lambda_{3}$, where $\lambda_{3} \equiv r\left(\Pi_{n}-c_{e}\right) /\left(2 c_{e}\right)$. The continuation payoff of an outsider is $O_{3}=\Pi_{n}-c_{e}$, whereas the continuation payoff of insiders is $I_{3}=\mu_{3}\left(\Pi_{n-3}-F\right)+\left(1-\mu_{3}\right) \Pi_{n}$, 
where $\mu_{3} \equiv r /\left(r+3 \lambda_{3}\right)$. Furthermore, these are the unique barrier equilibria if $c_{p} \geq c_{2}^{*}$.

(b) If $c_{p}<c_{2}^{*}$, there also exist barrier equilibria such that the three outsiders start playing a preemption game as soon as this subgame begins. In the continuous-time limit of the game, outsiders mix between $w$ and $p$. The continuation payoff of the outsiders converges to $O_{3}=\Pi_{n}-c_{e}$, whereas the continuation payoff of the insiders is $I_{3}=\phi_{3}(1) I_{2}+\left(1-\phi_{3}(1)\right) \Pi_{n}$, where $\phi_{3}(1)$ is the probability of a single outsider entering (out of the three existing ones). ${ }^{3}$

Proof. Consider first subgames with three outsiders in which insiders play a no-barrier equilibrium in the subgame with two outsiders left. As we showed in the proof of Lemma A (now for the case of $k=3$ ), there always exist a no-barrier equilibrium in the current subgame, and no other equilibrium exists if $F>\Pi_{n-3}-\Pi_{n}$. For $F<\Pi_{n-3}-\Pi_{n}$, there also exist barrier equilibria in the current subgame in which insiders pay $f$ with probability one and outsiders mix between actions $u$ and $w$. Each outsider chooses her entry time according to an exponential distribution with parameter $\lambda_{3} \equiv r\left(\Pi_{n}-c_{e}\right) /\left(2 c_{e}\right)$, so $O_{3}=\Pi_{n}-c_{e}$ and $I_{3}=\mu_{3}\left(\Pi_{n-3}-F\right)+\left(1-\mu_{3}\right) \Pi_{n}$, where $\mu_{3} \equiv r /\left(r+3 \lambda_{3}\right)$.

Consider now subgames with three outsiders in which insiders play the barrier equilibrium in the subgame with two outsiders left (which requires that $\left.F<\Pi_{n-2}-\Pi_{n}\right)$. Regardless of parameter values, there always exist a no-barrier equilibrium in the current subgame, so it simply remains to examine (and characterize if applicable) other types of equilibria.

Throughout the remainder of the proof, we normalize to zero the date at which the subgame with three outsiders starts. Recalling that each player in a mixed-strategy equilibrium should be indifferent among all actions chosen with positive probability, we have that outsiders may mix in principle using the three actions available to each of them, namely $w, p$ and $u$. Similarly, insiders may mix between $\widehat{p}$ and $\widehat{u}$. We denote $\rho_{a, k} \geq 0$ for the probability

\footnotetext{
${ }^{3}$ See expression (7) in the proof for the specific formula for $\phi_{3}(1)$.
} 
with which one of the outsiders plays action $a$ when $k$ outsiders remain to enter, whereas $\varphi_{k}>0$ denotes the probability with which each insider plays action $\widehat{p}$ when $k$ outsiders remain to enter (recall we have already analyzed the case in which $\varphi_{k}=0$ ). We let $V_{a, k}$ denote the outsider's payoff when following action $a \in\{w, p, u\}$ (given the mixing probabilities used by the other players), and denote the insider's payoff when following action $\widehat{a} \in\{\widehat{p}, \widehat{u}\}$ by $V_{\widehat{a}, k} \cdot{ }^{4}$ We have the following:

$$
\begin{aligned}
V_{p, 3}= & \rho_{w, 3}^{2}\left(\pi_{n-2} \Delta+I_{2} \delta\right)+2 \rho_{w, 3}\left(1-\rho_{w, 3}\right)\left(\pi_{n-1} \Delta+\Pi_{n} \delta\right)+\left(1-\rho_{w, 3}\right)^{2} \Pi_{n}-c_{e}-c_{p} \\
& -\left(1-\varphi_{3}^{n-3}\right) \rho_{w, 3}^{2}\left(I_{2}-\Pi_{n}\right) \delta \\
V_{u, 3}= & \rho_{w, 3}^{2}\left(\pi_{n-2} \Delta+\Pi_{n} \delta\right)+2 \rho_{w, 3}\left(1-\rho_{w, 3}\right)\left(\pi_{n-1} \Delta+\Pi_{n} \delta\right)+\left(1-\rho_{w, 3}\right)^{2} \Pi_{n}-c_{e}
\end{aligned}
$$

and

$$
\begin{aligned}
V_{w, 3}= & \varphi_{3}^{n-3}\left[\rho_{w, 3}^{2}\left(V_{w, 3} \delta\right)+2 \rho_{w, 3} \rho_{p, 3} O_{2} \delta+\left(\rho_{w, 3}+\rho_{p, 3}+1\right) \rho_{u, 3} \Pi_{n} \delta+\rho_{p, 3}^{2}\left(\Pi_{n}-c_{e}\right) \delta\right] \\
& +\left(1-\varphi_{3}^{n-3}\right) \Pi_{n} \delta
\end{aligned}
$$

Letting $C_{3}^{l}=\left(\begin{array}{l}3 \\ l\end{array}\right)$ denotes the binomial coefficient indexed by 3 and $l$, we can also compute what an insider expects to earn when not paying $f$,

$$
V_{\widehat{u}, 3}=\Pi_{n} \delta+\sum_{l=0}^{3} C_{3}^{l}\left(\rho_{w, 3}\right)^{l}\left(1-\rho_{w, 3}\right)^{3-l}\left(\pi_{n-l} \Delta\right),
$$

and similarly when she does pay $f$ :

$$
\begin{aligned}
V_{\widehat{p}, 3}= & \varphi_{3}^{n-4}\left\{\begin{array}{c}
\left(\rho_{w, 3}\right)^{3}\left(\pi_{n-3} \Delta+\widehat{V}_{p, 3} \delta\right)+3\left(\rho_{w, 3}\right)^{2}\left[\rho_{u, 3}\left(\pi_{n-2} \Delta+\Pi_{n} \delta\right)+\rho_{p, 3}\left(\pi_{n-2} \Delta+I_{2} \delta\right)\right]+ \\
3 \rho_{w, 3}\left[\left(\rho_{u, 3}\right)^{2}\left(\pi_{n-1} \Delta+\Pi_{n} \delta\right)+\left(\rho_{p, 3}\right)^{2}\left(\pi_{n-1} \Delta+\Pi_{n} \delta\right)+2 \rho_{u, 3} \rho_{p, 3}\left(\pi_{n-1} \Delta+\Pi_{n} \delta\right)\right] \\
+\left(1-\rho_{w, 3}\right)^{3}\left(\pi_{n} \Delta+\Pi_{n} \delta\right)
\end{array}\right\} \\
& +\left(1-\varphi_{3}^{n-4}\right)\left[\Pi_{n} \delta+\sum_{l=0}^{3} C_{3}^{l}\left(\rho_{w, 3}\right)^{l}\left(1-\rho_{w, 3}\right)^{3-l}\left(\pi_{n-l} \Delta\right)\right]-f \Delta .
\end{aligned}
$$

\footnotetext{
${ }^{4}$ All these probabilities $\left(\rho_{a, k}, a \in\{w, p, u\}\right.$, and $\left.\varphi_{k}\right)$ and value functions $\left(V_{a, k}, a \in\right.$ $\{w, p, u\}$, and $V_{\widehat{a}, k}, \widehat{a} \in\{\widehat{p}, \widehat{u}\}$ ) depend on $\Delta$, but we omit the dependence to avoid clutter.
} 
The facts that $\rho_{u, 3}+\rho_{p, 3}=1-\rho_{w, 3}$ and $\sum_{l=0}^{3} C_{3}^{l}\left(\rho_{w, 3}\right)^{l}\left(1-\rho_{w, 3}\right)^{3-l}=1$ yield that the expression for $V_{\widehat{p}, 3}$ can be rewritten after some manipulations as

$$
V_{\widehat{p}, 3}=\Pi_{n}+\frac{3 \varphi_{3}^{n-4}\left(\rho_{w, 3}\right)^{2} \rho_{p, 3} \Pi_{n}+\Pi_{n}(\delta-1)+\sum_{l=0}^{3} C_{3}^{l}\left(\rho_{w, 3}\right)^{l}\left(1-\rho_{w, 3}\right)^{3-l}\left(\pi_{n-l} \Delta\right)-f \Delta}{1-\varphi_{3}^{n-4}\left(\rho_{w, 3}\right)^{3} \delta} .
$$

Note first that action $p$ is dominated (i.e., $\rho_{p, 3}=0$ ) when $c_{p} \geq c_{2, F}^{*}$, so the argument used in the proof of Lemma A (for the case of $k=3$ ) proves part 2 a. ${ }^{5}$ To complete the proof, it simply remains to analyze the cases in which $c_{p}<c_{2, F}^{*}$. In these cases, we must have $\rho_{p, 3}>0$ : otherwise, outsiders would engage in a waiting game such as the one studied in the proof of Lemma $\mathrm{A}$ (for $k=3$ ), and $c_{p}<c_{2, F}^{*}$ would imply that an outsider could do better off by choosing action $p$ at $\tau=0$. Outsiders must clearly always put positive weight on $w$, since they wish to avoid coordination failures when entering if there is an incentive to preempt, and they wish to free ride on others if there is some incentive to enter without protection. So we must also have $\rho_{w, 3}>0$ (otherwise, we would have $V_{u, 3}>V_{p, 3}$, which would contradict $\rho_{p, 3}>0$ ). So both $\rho_{p, 3}$ and $\rho_{w, 3}$ must be bounded away from 0 whenever $c_{p}<c_{2, F}^{*}$.

We now show that we must have $V_{\widehat{u}, 3}<V_{\widehat{p}, 3}$ for $\Delta>0$ small enough, which means that $\varphi_{3}$ must equal 1 in the continuous-time limit of the game. To demonstrate this result, note that $V_{\widehat{u}, 3}<V_{\widehat{p}, 3}$ if and only if

$3 \varphi_{3}^{n-4}\left(\rho_{w, 3}\right)^{2} \rho_{p, 3} \Pi_{n}+\varphi_{3}^{n-4}\left(\rho_{w, 3}\right)^{3} \delta\left[\Pi_{n}(\delta-1)+\sum_{l=0}^{3} C_{3}^{l}\left(\rho_{w, 3}\right)^{l}\left(1-\rho_{w, 3}\right)^{3-l}\left(\pi_{n-l} \Delta\right)\right]>f \Delta$,

so the facts that $\delta \approx 1-r \Delta$ and $\varphi_{3} \Pi_{n}>0$ (recall the hypothesis that $\left.\varphi_{3}>0\right)$ yields that the inequality holds for $\Delta>0$ small enough provided $\lim _{\Delta \rightarrow 0} \rho_{w, 3} \rho_{p, 3}>0$, which was shown earlier. So situations in which mixing by outsiders involves positive weights on $w$ and $p$ must be accompanied by insiders choosing to protect themselves: an insider finds it unilaterally optimal to choose $\varphi_{3}=1$ if the other insiders choose $\varphi_{3}=1$ as well.

\footnotetext{
${ }^{5}$ Note that playing barrier equilibria in the subgame with two outsiders left necessarily requires that $F<\Pi_{n-2}-\Pi_{n}$, so the fact that $c_{p}>0 \geq c_{2, F}^{*}$ for $F \geq \Pi_{n-2}-\Pi_{n}$ indeed shows that we have completed the proof of part 2a.
} 
To sum up the situation when $c_{p}<c_{2, F}^{*}$, we have shown that $\rho_{p, 3} \rho_{w, 3}>0$ and $\varphi_{3}=1$. We now prove by contradiction that action $u$ must be chosen with positive probability (even if turns out to be negligible as $\Delta \rightarrow 0$ ). Suppose to the contrary that $\rho_{u, 3}=0$. Then it is straightforward to show that $V_{p, 3}=V_{w, 3}$ yields $\rho_{w, 3} \rightarrow \sqrt{\frac{c_{p}}{I_{2}-\Pi_{n}}}$ as $\Delta \rightarrow 0$. Letting $V_{3}\left(\rho_{w, 3}, \Delta\right) \equiv V_{w, 3}-V_{p, 3}$, that is,

$$
\begin{aligned}
V_{3}\left(\rho_{w, 3}, \Delta\right)= & \frac{\left(1-\rho_{w, 3}^{2}\right)\left(\Pi_{n}-c_{e}\right) e^{-r \Delta}}{1-\rho_{w, 3}^{2} e^{-r \Delta}}-\rho_{w, 3}^{2}\left(\pi_{n-2} \Delta+I_{2} e^{-r \Delta}\right)+ \\
& 2 \rho_{w, 3}\left(1-\rho_{w, 3}\right)\left(\pi_{n-1} \Delta+\Pi_{n} e^{-r \Delta}\right)+\left(1-\rho_{w, 3}\right)^{2} \Pi_{n}-c_{e}-c_{p},
\end{aligned}
$$

the implicit function theorem implies that

$$
\begin{aligned}
\left.\frac{d \rho_{w, 3}}{d \Delta}\right|_{\Delta=0}= & -\frac{\left.\frac{\partial V_{3}}{\partial \Delta}\right|_{\Delta=0}}{\left.\frac{\partial V_{3}}{\partial \rho_{w, 3}}\right|_{\Delta=0}} \\
= & -\frac{\frac{r\left(\Pi_{n}-c_{e}\right)}{1-\rho_{w, 3}^{2}}+r \rho_{w, 3}\left(2\left(1-\rho_{w, 3}\right)\left(\Pi_{n-1}-\Pi_{n}\right)+\rho_{w, 3}\left(\Pi_{n-2}-I_{2}\right)\right)}{2 \rho_{w, 3}\left(I_{2}-\Pi_{n}\right)},
\end{aligned}
$$

so $\Pi_{n}<I_{2}<\Pi_{n-2}$ and $c_{e}<\Pi_{n}<\Pi_{n-1}$ imply that $\left.\frac{d \rho_{w, 3}}{d \Delta}\right|_{\Delta=0}<0$, which in turn implies that $\rho_{w, 3} \uparrow \sqrt{\frac{c_{p}}{I_{2}-\Pi_{n}}}$ as $\Delta \rightarrow 0$. Now consider an outsider who contemplates choosing action $u$ instead of $p$, noting that expressions (1) and (2) for $\varphi_{3}=1$ yield

$$
V_{u, 3}-V_{p, 3}=c_{p}-\delta \rho_{w, 3}^{2}\left(I_{2}-\Pi_{n}\right)
$$

Since $V_{u, 3}=V_{p, 3}$ for $\Delta=0$ (note $\rho_{w, 3}=\sqrt{\frac{c_{p}}{I_{2}-\Pi_{n}}}$ in such a case), it holds as $\Delta$ is infinitesimally increased that both $\delta$ and $\rho_{w, 3}^{2}$ decrease, so we would have that $V_{u, 3}-V_{p, 3}$ increases and hence $V_{u, 3}$ must be greater than $V_{p, 3}$ for $\Delta$ close enough to 0 . Because there can be no profitable deviation in equilibrium, we 
have found a contradiction, and therefore we must have that action $u$ is played with positive probability in a mixed-strategy equilibrium.

So we must have $V_{w, 3}=V_{p, 3}=V_{u, 3}$ in a mixed-strategy equilibrium in which players use stationary strategies. Since $V_{p, 3}=V_{u, 3}, \rho_{w, 3}>0$ yields

$$
\rho_{w, 3}=\sqrt{\frac{c_{p}}{\left(I_{2}-\Pi_{n}\right) \delta}}
$$

where we have used equated $V_{u, 3}-V_{p, 3}$ to 0 in expression (4). Using the working hypothesis that $c_{p}<c_{2, F}^{*} \equiv I_{2}-\Pi_{n}$ yields that $\frac{c_{p}}{\left(I_{2}-\Pi_{n}\right) \delta}<\delta^{-1}$, so $\rho_{w, 3}<1$ for $\Delta>0$ close enough to zero.

Because $\rho_{u, 3}=1-\left(\rho_{w, 3}+\rho_{p, 3}\right)$ and $O_{2}=\Pi_{n}-c_{e}$, the expression for $V_{w, 3}$ in (3) can be rewritten for $\varphi_{3}=1$ as follows:

$$
V_{w, 3}=\frac{\left(1-\rho_{w, 3}^{2}\right) \Pi_{n}-\rho_{p, 3}\left(\rho_{p, 3}+2 \rho_{w, 3}\right) c_{e}}{\delta^{-1}-\rho_{w, 3}^{2}} .
$$

Equating $V_{u, 3}$ and $V_{w, 3}$ yields the value for $\rho_{p, 3}>0$ after some manipulations:

$$
\rho_{p, 3}=\sqrt{\frac{c_{e}-(1-\delta) B \Pi_{n}-\Delta \rho_{w, 3}\left(1-\delta \rho_{w, 3}^{2}\right) C}{\delta c_{e}}}-\rho_{w, 3},
$$

where $B \equiv \delta\left(2-\rho_{w, 3}\right) \rho_{w, 3}^{3}+\left(1-\rho_{w, 3}\right)^{2}$ and $C \equiv 2 \pi_{n-1}\left(1-\rho_{w, 3}\right)+\rho_{w, 3} \pi_{n-2}$.

Using the fact that $I_{2}-\Pi_{n}=\mu_{2}\left(\Pi_{n-2}-\Pi_{n}-F\right)$, we find for small $\Delta>0$ that

$$
\begin{gathered}
\rho_{w, 3} \approx \sqrt{\frac{c_{p}}{\mu_{2}\left(\Pi_{n-2}-\Pi_{n}-F\right)}}, \\
\rho_{p, 3} \approx 1-\sqrt{\frac{c_{p}}{\mu_{2}\left(\Pi_{n-2}-\Pi_{n}-F\right)}},
\end{gathered}
$$

and

$$
\rho_{u, 3} \approx 0
$$

that is, action $u$ is played with positive but vanishing probability as $\Delta \rightarrow 0$.

We now determine payoffs. Given $m$ periods of play between time 0 and some fixed time $\tau>0$, it holds that the probability that no outsider has 
entered and paid for protection once time $\tau$ arrives is $\left(\rho_{w, 3}\right)^{3 m}=\left(\rho_{w, 3}\right)^{3 \tau / \Delta}$ (since $m=\tau / \Delta)$, which converges to zero as $\Delta$ converges to zero for any arbitrarily chosen $\tau>0$. We then must have that there is probability one that at least one outsider will enter and pay for protection (almost) instantaneously. In words, outsiders correlate their actions as $\Delta$ goes to zero even though they randomize independently.

We conclude the proof by characterizing the probability distribution over entry outcomes at (normalized) time 0 as well as equilibrium payoffs for the case under consideration. Because the probability of no entry at any point in time is $\left(1-\rho_{p, 3}\right)^{3}$, it holds that the probability that at least one outsider enters is $1-\left(1-\rho_{p, 3}\right)^{3}$. Conditional upon at least one outsider entering, we then have that

$$
\begin{gathered}
\phi_{3}(3)=\left(\rho_{p, 3}\right)^{3} /\left(1-\left(1-\rho_{p, 3}\right)^{3}\right), \\
\phi_{3}(2)=3\left(1-\rho_{p, 3}\right)\left(\rho_{p, 3}\right)^{2} /\left(1-\left(1-\rho_{p, 3}\right)^{3}\right),
\end{gathered}
$$

and

$$
\phi_{3}(1)=3\left(1-\rho_{p, 3}\right)^{2} \rho_{p, 3} /\left(1-\left(1-\rho_{p, 3}\right)^{3}\right) \text {, }
$$

where $\phi_{k}(l)$ denotes the probability that $l \geq 1$ outsiders enter simultaneously at $\tau=0$ given that there are $k \geq l$ of them. We finally observe that an outsider's continuation payoff at the beginning of these subgames is $O_{3}=\Pi_{n}-c_{e}$ (since $V_{p, 3}=V_{u, 3}=V_{w, 3} \approx \Pi_{n}-c_{e}$ for small enough $\Delta>0$ ). Since $I_{1}=I_{0}=\Pi_{n}$, the expected payoff earned by an insider is

$$
I_{3}=\phi_{3}(1) I_{2}+\left(1-\phi_{3}(1)\right) \Pi_{n}
$$

More than three outsiders left to enter

The ideas uncovered in the subgames with three outsiders partially extend to the subgames with a larger number of outsiders. ${ }^{6}$ Letting $c_{k, F}^{*} \equiv \mu_{k}\left(\Pi_{n-k}-\right.$

\footnotetext{
${ }^{6}$ We recall that if a no-barrier (continuation) equilibrium is played in a subgame with $k$ outsiders, then no-barrier equilibria are assumed to be played in all subgames with less
} 
$\Pi_{n}-F$ ), it holds in the subgames with $k \geq 3$ outsiders that $c_{p} \geq c_{k-1, F}^{*}$ implies that the only barrier equilibrium is such that players mix between $w$ and $u$, and the time of first entry is exponentially distributed. As in the paper's main text, the critical ingredient of the induction argument is that $\left\{c_{k, F}^{*}\right\}_{k=2}^{n-1}$ is a monotonically increasing sequence. ${ }^{7}$ This implies that, when $c_{p} \geq c_{k-1, F}^{*}$, if one outsider chooses to enter by paying the protection cost $c_{p}$, the $k-1$ remaining outsiders will then engage in a waiting game, since $c_{p}>c_{k-2, F}^{*}$. The following result therefore holds.

Lemma C In subgames with $k \in\{3, \ldots, n-1\}$ outsiders:

1. There always exists a no-barrier continuation equilibrium such that insiders never pay $f$ and outsiders choose action $u$. If $F>\Pi_{n-k}-\Pi_{n}$, this is the unique (symmetric) equilibrium.

2. If $F<\Pi_{n-k}-\Pi_{n}$, there also exists a barrier continuation equilibrium such that insiders pay $f$ while the subgame is not over and the $k$ outsiders mix between actions $u$ and $w$. In the continuous-time limit of the game, the entry time of outsiders is drawn from an exponential distribution with parameter $k \lambda_{k}$, where $\lambda_{k} \equiv r\left(\Pi_{n}-c_{e}\right) /\left((k-1) c_{e}\right)$. The continuation payoff of an outsider is $O_{k}=\Pi_{n}-c_{e}$, whereas the continuation payoff of an insider is $I_{k}=\mu_{k}\left(\Pi_{n-k}-F\right)+\left(1-\mu_{k}\right) \Pi_{n}$, where $\mu_{k} \equiv r /\left(r+k \lambda_{k}\right)$. If $c_{p} \geq c_{k-1, F}^{*}$, this is the unique barrier equilibrium.

Proof. We prove the result by induction. Lemma B established the result for $k=3$, so it only remains to prove that it holds for $k \geq 4$ whenever it is true for $k-1$. So suppose that the result holds for $k-1$, and consider the subgames with $k$ outsiders when $c_{p} \geq c_{k-1}^{*}$. Let us focus on an outsider's incentive to play $p$. Since $c_{k^{\prime}, F}^{*}<c_{k-1, F}^{*}$ for all $k^{\prime}<k-1$, she knows when choosing action

than $k$ outsiders.

${ }^{7}$ Note that $\mu_{k}=(k-1) c_{e} /\left(k \Pi_{n}-c_{e}\right)$ is increasing in $k$, since $c_{e}<\Pi_{n}$ implies that $d \mu_{k} / d k=c_{e}\left(\Pi_{n}-c_{e}\right) /\left(k \Pi_{n}-c_{e}\right)^{2}>0$. Taking into account that both $\mu_{k}$ and $\Pi_{n-k}-\Pi_{n}-F$ are positive, the fact that $\Pi_{n-k}$ and $\mu_{k}$ are both increasing in $k$ then yields that $c_{2, F}^{*}<$ $c_{3, F}^{*}<\ldots<c_{n-1, F}^{*}$. 
$p$ that $p$ being simultaneously chosen by $l \geq 0$ other outsiders will result in the remaining ones playing a waiting game (by the induction hypothesis). Clearly, the highest payoff that can be achieved is the one attained when no other outsider enters simultaneously, i.e., when $l=0$. Thus, the highest payoff she can obtain by taking action $p$ is $I_{k-1}-c_{p}-c_{e}=\mu_{k-1} \Pi_{n-k+1}+\left(1-\mu_{k-1}\right) \Pi_{n}-$ $c_{p}-c_{e}$. Since $c_{p} \geq c_{k-1, F}^{*}$ implies $I_{k-1}-c_{p}-c_{e}<\Pi_{n}-c_{e}$, it then follows that no outsider must be willing to enter by paying the protection cost in subgames with $k$ outsiders. Letting $k \geq 4$ in the proof of Lemma A then delivers the desired result.

We now consider the more complex case where preemption can occur. It is essential for our purposes to define $J^{\prime}$, the critical number of outsiders such that a waiting game is played if the number of outsiders is strictly less than $J^{\prime}$ (i.e., in subgames in which the number of outsiders left to enter equals $2, \ldots, J^{\prime}-1$ ). As we explained earlier, outsiders can go from playing a preemption game to a waiting game for two reasons: either it is expected that a no-barrier equilibrium will be played in the next subgame, or, even if it is expected that a barrier equilibrium will then be played, it is still not worthwhile to pay for protection upon entry $\left(c_{p}>c_{k-1, F}^{*}\right)$. Corresponding to the first case, we define $J_{1}$ as the maximum value of $k$ such that the continuation equilibrium played in a subgame with $k-1$ outsiders is of the no-barrier type, so $J_{1}$ ranges from 2 to $n$. For the second case, we define $J_{2} \equiv \inf \left\{k \geq 3: c_{p}<c_{k-1, F}^{*}\right\}$, where $J_{2}=n$ if it is not well defined. Note that $J_{2}$ is a step function of $c_{p}$ ranging from 3 to $n$. Overall, we let $J^{\prime} \equiv \max \left(J_{1}, J_{2}\right){ }^{8}$ As soon as any of these thresholds $J_{1}$ and $J_{2}$ is crossed, a waiting game starts. We will now show that for $k \geq J^{\prime}$, a series of preemption games takes place. A priori, the players may mix between the three available actions, $w, p$ and $u$. We can show that action $u$ is chosen, but with vanishing probability as $\Delta$ goes to zero, so we have the following result.

Lemma D In subgames with $k \in\left\{J^{\prime}, \ldots, n-1\right\}$ outsiders such that $c_{p}<$ $c_{k-1, F}^{*}$, the unique symmetric equilibrium in the continuous-time limit of the

\footnotetext{
${ }^{8}$ Note that $J=J_{2}$, so $J^{\prime} \geq J$, and hence the introduction of a flow cost of protection mitigates preemption incentives whenever $J_{1}>J_{2}$.
} 
game has the following features: ${ }^{9}$

(i) Outsiders mix only between actions $p$ and $w$ at each point in time, and the probability $\rho_{k}$ of playing $p$ is uniquely determined.

(ii) Such probability $\rho_{k}$ increases with $k$.

(iii) Immediate entry by at least one outsider occurs.

Proof. The current situation is significantly more complex than the one we analyzed earlier when $k=3$, since a sequence of preemption games can now occur. In the three outsider case, there was at most a single preemption game being played and we could thus derive probabilities explicitly and take the limit for small values of $\Delta$. When $k>3$, the probabilities of entry in each subgame exhibiting preemption features must be determined recursively, so we shall pursue the following approach for characterizing the game's continuoustime limit. For a given period length $\Delta$, let $\rho_{a, k}(\Delta) \geq 0$ be the probability with which each outsider plays action $a \in\{w, p, u\}$ when $k$ outsiders are left to enter. Also, let $\rho_{\widehat{a}, k}(\Delta) \geq 0$ be the probability with which each insider plays action $\widehat{a} \in\{\widehat{p}, \widehat{u}\}$ in such subgames. Furthermore, let $V_{a, k}(\Delta)$ denote the outsider's payoff from choosing action $a$ given that the $k-1$ other outsiders are mixing over actions with probability $\rho_{a, k}(\Delta)$ and the $n-k$ insiders choose $\rho_{\widehat{a}, k}(\Delta)$. In equilibrium, the mixing probabilities $\rho_{a, k}(\Delta)$ for $a \in\{w, p, u\}$ must be such that outsiders are indifferent between all three actions and such that these are indeed probabilities (i.e. $V_{p, k}(\Delta)=V_{u, k}(\Delta)=V_{w, k}(\Delta), \rho_{a, k}(\Delta) \in$ $(0,1)$ and $\left.\sum_{a \in\{w, p, u\}} \rho_{a, k}(\Delta)=1\right)$. In addition, $\rho_{\widehat{p}, k}(\Delta)=1$ requires that $V_{\widehat{p}, k}(\Delta)>V_{\widehat{u}, k}(\Delta)$, whereas $\rho_{\widehat{u}, k}(\Delta)=1$ requires that $V_{\widehat{p}, k}(\Delta)<V_{\widehat{u}, k}(\Delta)$.

\footnotetext{
${ }^{9}$ The proof of the lemma also reveals when $J^{\prime} \neq n$ that $I_{n-1}<I_{n-2}<\ldots<I_{J^{\prime}}<I_{J^{\prime}-1}$, i.e., the gate-keeper expects her payoff to improve as preemption by outsiders choosing protected entry takes place: even though entry harms the gate-keeper's payoff, the threat of potential entry becomes less intense because the remaining outsiders enter with a smaller probability, and the latter effect dominates the former. The finding that $I_{n-1}<I_{n-2}<$ $\ldots<I_{J^{\prime}}<I_{J^{\prime}-1}$ when $J^{\prime} \neq n$, coupled with our previous one that $I_{J^{\prime}-1}>I_{J^{\prime}-2}>\ldots>$ $I_{0}=\Pi_{n}$ (see previous lemma), means that the gate-keeper's expected payoff peaks when the outsiders' preemption incentives vanish and their incentives to free-ride on each other appear (see Vettas 2000 for the discovery of such payoff nonmonotonicity result in a related context.). When $J^{\prime}=n$, it always holds that $I_{J^{\prime}-1}>I_{J^{\prime}-2}>\ldots>I_{0}=\Pi_{n}$, so the gatekeeper is severely harmed by outsider entry (her expected payoff falls from $I_{J^{\prime}-1}$ to $I_{0}=\Pi_{n}$ as soon as there is entry).
} 
What we will do is to prove for small $\Delta>0$ that either $V_{\widehat{p}, k}(\Delta)>V_{\widehat{u}, k}(\Delta)$ or $V_{\widehat{p}, k}(\Delta)<V_{\widehat{u}, k}(\Delta)$ and then solve for the solution of the system consisting of $V_{p, k}(\Delta)=V_{u, k}(\Delta)=V_{w, k}(\Delta)$ for $\Delta=0$, what we call the continuous-time approximation of the equilibrium. ${ }^{10}$ We will show that this solution exists and is unique, so that the fact that the value functions $V_{a, k}(\Delta)(a \in\{w, p, u\})$ are continuous in $\Delta$ and in the probabilities will imply that our approach effectively provides the limit of equilibrium play when we move to continuous time.

To illustrate further this method, consider the case of three players solved in the proof of Lemma B. In that case we solved explicitly, for a small fixed value of $\Delta$, for the probabilities $\rho_{a, 3}(\Delta), a \in\{w, p, u\}$ (see (5) and (6)). We see from the solution presented in the proof of Lemma $B$, that taking the limit of all the probabilities as $\Delta$ converges to zero (as we did) leads to the same solution as directly solving the system consisting of equations (1)-(3) for $\Delta=0$, as was to be expected due to the continuity of the system. From now on, $\rho_{a, k}$ and $V_{a, k}$ shall respectively denote $\rho_{a, k}(\Delta)$ and $V_{a, k}(\Delta)$ for $\Delta=0$.

In order to solve for the approximation of the equilibrium outcome, we work directly with the solution for $\Delta=0$. As in the proof of Lemma $\mathrm{B}$, it is easy to show that insiders cannot be randomizing between $\widehat{u}$ and $\widehat{p}$, whereas outsiders must be choosing each of the three actions available to them with positive probability. Taking this into account, we prove the result in a number of steps (the proof is quite similar to the one in the paper's appendix).

Step 1: $\rho_{u, k}=0 .{ }^{11}$

We show this result by induction. For $\Delta=0$, we have

$$
V_{u, J^{\prime}}=\Pi_{n}-c_{e}
$$

\footnotetext{
${ }^{10}$ Formally, what we mean by (continuous-time) approximation of the equilibrium is a set of admissible mixing probabilities $\rho_{a, k}(a \in\{p, u, w\})$ satisfying the following property: for any (small) $\epsilon>0$, there exists $\Delta_{\epsilon}$ such that $\Delta<\Delta_{\epsilon}$ implies that $\left|\rho_{a, k}(\Delta)-\rho_{a, k}\right|<\epsilon$, where $\rho_{a . k}(\Delta)$ is the exact equilibrium play.

${ }^{11}$ Rigorously speaking, this should be interpreted as $\rho_{u, k} \downarrow 0$ as $\Delta \rightarrow 0$.
} 
and

$$
\begin{aligned}
V_{w, J^{\prime}}= & \operatorname{Pr}\left[X_{w, J^{\prime}}=J^{\prime}-1, X_{p, J^{\prime}}=0, X_{u, J^{\prime}}=0\right] V_{w, J^{\prime}}+ \\
& \sum_{m=1} \sum_{l=0}^{J^{\prime}-1} \operatorname{Pr}\left[X_{w, J^{\prime}}=J^{\prime}-1-l-m, X_{p, J^{\prime}}=l, X_{u, J^{\prime}}=m\right] \Pi_{n}+ \\
& \sum_{l=1}^{J^{\prime}-1} \operatorname{Pr}\left[X_{w, J^{\prime}}=J^{\prime}-1-l, X_{p, J^{\prime}}=l, X_{u, J^{\prime}}=0\right] O_{J^{\prime}-l},
\end{aligned}
$$

where $\operatorname{Pr}\left[X_{w, k}, X_{p, k}, X_{u, k}\right]$ denotes the probability that $X_{w, k}$ outsiders choose $w, X_{p, k}$ outsiders choose $p$ and $X_{u, k}$ outsiders choose $u$. We know, that for all $k<J^{\prime}$, a waiting game is played and, according to Lemma C, $O_{k}=\Pi_{n}-c_{e}$, so the system of equations can be rewritten as

$$
V_{u, J^{\prime}}=\Pi_{n}-c_{e}
$$

and

$$
\begin{aligned}
V_{w, J^{\prime}}= & \operatorname{Pr}\left[X_{w, J^{\prime}}=J^{\prime}-1, X_{p, J^{\prime}}=0, X_{u, J^{\prime}}=0\right] V_{w, J^{\prime}}+ \\
& \left(1-\operatorname{Pr}\left[X_{w, J^{\prime}}=J^{\prime}-1, X_{p, J^{\prime}}=0, X_{u, J^{\prime}}=0\right]\right) \Pi_{n}- \\
& \sum_{l=1}^{J^{\prime}-1} \operatorname{Pr}\left[X_{w, J^{\prime}}=J^{\prime}-1-l, X_{p, J^{\prime}}=l, X_{u, J^{\prime}}=0\right] c_{e} .
\end{aligned}
$$

In a mixed-strategy equilibrium, an outsider must be indifferent between all actions played with positive probability, so we must have $V_{u, J^{\prime}}=V_{w, J^{\prime}}$, which implies that

$\sum_{l=1}^{J^{\prime}-1} \operatorname{Pr}\left[X_{w, J^{\prime}}=J^{\prime}-1-l, X_{p, J^{\prime}}=l, X_{u, J^{\prime}}=0\right] /\left(1-\operatorname{Pr}\left[X_{w, J^{\prime}}=J^{\prime}-1, X_{p, J^{\prime}}=0, X_{u, J^{\prime}}=0\right]\right)=1$.

This holds if and only if

$$
\sum_{l=0}^{J^{\prime}-1} \operatorname{Pr}\left[X_{w, J^{\prime}}=J^{\prime}-1-l, X_{p, J^{\prime}}=l, X_{u, J^{\prime}}=0\right]=1
$$


hence we get that $\rho_{u, J^{\prime}}=0$. Further, this implies that $O_{J^{\prime}}=\Pi_{n}-c_{e}$, and the property is therefore true for $k=J^{\prime}$. The reasoning follows exactly the same lines for larger values of $k$ (since $u$ is chosen in equilibrium, but with vanishing probability as $\Delta \rightarrow 0$ ).

Thus, in the approximation we consider, the players will essentially mix just between actions $w$ and $p$ in subgames in which insiders pay $f$ while the subgame is not over. We denote $\rho_{k} \equiv \rho_{p, k}$ for the individual probability of entry (so we have $\rho_{w, k}=1-\rho_{k}$ ). Given $k$ outsiders, the payoff to choosing action $p$ is given by

$$
V_{p, k}=\sum_{l=0}^{k-1} C_{k-1}^{l}\left(\rho_{k}\right)^{l}\left(1-\rho_{k}\right)^{k-1-l} I_{k-1-l}-c_{p}-c_{e}
$$

where $C_{k-1}^{l}=\left(\begin{array}{c}k-1 \\ l\end{array}\right)$ denotes the binomial coefficient indexed by $k-1$ and $l$. The value to an outsider of paying the protection cost when entering depends on how many other outsiders simultaneously enter. If $l$ other outsiders enter, the outsider participates in the next period as an insider in a subgame with $k-1-l$ outsiders. Her expected gain in this case is thus $I_{k-1-l}$ (the continuation value of being an insider with $k-1-l$ outsiders).

Each of the $k$ outsiders will mix between $p$ and $w$ so as to leave others indifferent between these two actions, which yields that

$$
V_{p, k}=\Pi_{n}-c_{e}
$$

since $V_{w, k}=V_{u, k}=\Pi_{n}-c_{e}$ for $\Delta=0$. Letting $\bar{I}_{k-1-l} \equiv I_{k-1-l}-\Pi_{n}$ and

$$
F_{k}(\rho) \equiv \sum_{l=0}^{k-1} C_{k-1}^{l} \rho^{l}(1-\rho)^{k-1-l} \bar{I}_{k-1-l}
$$

the indifference condition can be equivalently written as:

$$
F_{k}\left(\rho_{k}\right)=c_{p}
$$

Step 2: $\rho_{k}$ is the unique solution to $F_{k}\left(\rho_{k}\right)=c_{p}$. 
Having established in Step 1 that $\rho_{k}$ must necessarily solve $F_{k}\left(\rho_{k}\right)=c_{p}$, we now show that there exists a unique solution to such an equation. Consider first the last "preemption game," i.e., the subgame where $J^{\prime}$ outsiders are left to enter. As shown in the main text, the indifference between actions $p$ and $w$ is defined by

$$
F_{J^{\prime}}\left(\rho_{J^{\prime}}\right)=c_{p}
$$

where

$$
F_{J^{\prime}}(\rho)=\sum_{l=0}^{J^{\prime}-1} C_{J^{\prime}-1}^{l} \rho^{l}(1-\rho)^{J^{\prime}-1-l} \bar{I}_{J^{\prime}-1-l} .
$$

Note that following entry by at least one outsider, a waiting game is played (the speed will be determined by the number of other outsiders who happen to enter). Note that according to Lemma C, $\bar{I}_{J^{\prime}-1-l}=\mu_{J^{\prime}-1-l}\left(\Pi_{n-\left(J^{\prime}-1-l\right)}-\Pi_{n}\right)=$ $c_{J^{\prime}-1-l, F}^{*}$. We showed previously that $c_{k, F}^{*}$ is an increasing function of $k$. So we have $\bar{I}_{J^{\prime}-1}>\bar{I}_{J^{\prime}-2}>\ldots>\bar{I}_{0}$, and it can be immediately observed that $F_{J^{\prime}}(\rho)$ strictly decreases with $\rho$. Indeed, increasing $\rho$ shifts the distribution to states with lower payoffs.

Furthermore, if $J^{\prime}=J_{2}$, then we have $F_{J_{2}}(0)=\bar{I}_{J_{2}-1}=c_{J_{2}-1, F}^{*}>c_{p}$ (since $J_{2}=\inf \left\{k \geq 3: c_{p}<c_{k-1, F}^{*}\right\}$ by definition). If instead $J^{\prime}=J_{1}$, we also have $F_{J_{1}}(0)=\bar{I}_{J_{1}-1}=c_{J_{1}-1, F}^{*}>c_{p}$ (since $J_{1} \geq J_{2}$ implies $c_{p}<c_{J_{2}-1, F}^{*} \leq c_{J_{1}-1, F}^{*}$ by the increasingness of $\left.c_{k, F}^{*}\right)$. So it always holds that $F_{J^{\prime}}(0)=\bar{I}_{J^{\prime}-1}=c_{J^{\prime}-1, F}^{*}>$ $c_{p}$. Since $F_{J^{\prime}}(1)=\bar{I}_{0}=0$ and $F_{J^{\prime}}(\rho)$ is a continuous and strictly decreasing function, it then follows that the equation $F_{J^{\prime}}(\rho)=c_{p}$ has a unique solution $\rho_{J^{\prime}} \in(0,1)$.

We now work recursively with $F_{k+1}(\rho)$ for $k \geq J^{\prime}$. We use the following key properties of $F_{k+1}(\rho)$ proven below:

- Property 1:

$$
\frac{\partial F_{k+1}}{\partial \rho}(\rho)=\left(\frac{k}{1-\rho}\right)\left(F_{k}(\rho)-F_{k+1}(\rho)\right) .
$$

- Property 2:

$$
\frac{\partial F_{k+1}}{\partial \rho}(0)>0
$$


- Property 3:

$$
\bar{I}_{k}=F_{k+1}\left(\rho_{k}\right) .
$$

From Properties 1 and 2, we can show recursively that $F_{k+1}(\rho)$ is increasing at zero, reaches a maximum when $F_{k+1}(\rho)$ and $F_{k}(\rho)$ cross and is then decreasing. ${ }^{12}$ Furthermore, we know that $F_{k+1}(1)=\bar{I}_{0}=0$. So to establish that $F_{k+1}(\rho)=c_{p}$ has a unique solution it is sufficient to show that $F_{k+1}(0)>c_{p}$. To prove it, note that we have $F_{k+1}(0)=\bar{I}_{k}$, and Property 3 implies that $\bar{I}_{k}=F_{k+1}\left(\rho_{k}\right)$, so it holds that $F_{k+1}(0)=F_{k+1}\left(\rho_{k}\right)$. Because $F_{k+1}(\rho)$ is increasing at zero according to Property 2 , the unique maximum must be reached somewhere between 0 and $\rho_{k}$. According to Property 1, we know that $F_{k+1}(\rho)>F_{k}(\rho)$ for $\rho \geq \rho_{k}$, and therefore $F_{k+1}\left(\rho_{k}\right)>F_{k}\left(\rho_{k}\right)$. Taking into account that $F_{k+1}(0)=F_{k+1}\left(\rho_{k}\right)$, as we just showed, and that $F_{k}\left(\rho_{k}\right)=c_{p}$, it follows that $F_{k+1}(0)>c_{p}$.

Step 3: Lemma D (i) follows directly from steps 1 and 2. We also showed above that $F_{k+1}(\rho)>c_{p}$ for $\rho \in\left(0, \rho_{k}\right)$, so we must that have $\rho_{k}<\rho_{k+1}$, which proves (ii). Finally, (iii) can be shown as in the proof of Lemma B.

To conclude the proof, we show that properties 1-3 stated above do hold:

Property 1 We have that

$$
F_{k}(\rho)=\sum_{l=0}^{k-1} C_{k-1}^{l}(\rho)^{l}(1-\rho)^{k-1-l} \bar{I}_{k-1-l}
$$

and

$$
F_{k+1}(\rho)=\sum_{l=0}^{k} C_{k}^{l}(\rho)^{l}(1-\rho)^{k-l} \bar{I}_{k-l} .
$$

\footnotetext{
${ }^{12}$ Indeed, we have shown that $F_{J^{\prime}}$ is a decreasing function. By Property $2, F_{J^{\prime}+1}$ is increasing at zero and must cross $F_{J^{\prime}}$ at least once since $F_{J^{\prime}}$ converges to zero when $\rho$ converges to one and $F_{J^{\prime}+1}$ starts above zero. At this intersection point, by Property 1 , $F_{J^{\prime}+1}$ must reach a local maximum. Such a function cannot have another turning point (which should be a local minimum given that $F_{J^{\prime}+1}$ is continuously differentiable): if this were the case, $F_{J^{\prime}+1}$ should be decreasing at a faster rate than the everywhere decreasing function $F_{J^{\prime}}$, so the continuous differentiability of $F_{J^{\prime}+1}$ rules out that $F_{J^{\prime}+1}$ be tangent to $F_{J^{\prime}}$ at the local minimum, and it must be that $F_{J^{\prime}+1}$ and $F_{J^{\prime}}$ cross at such a point, which in turn would contradict Property 1 applied on the right neighborhood of the turning point by the continuous differentiability of $F_{J^{\prime}+1}$.
} 
So we can establish that

$$
\begin{aligned}
F_{k}(\rho)-F_{k+1}(\rho) & =\sum_{l=0}^{k-1} C_{k-1}^{l}(\rho)^{l}(1-\rho)^{k-1-l} \bar{I}_{k-1-l}-\sum_{l=0}^{k} C_{k}^{l}(\rho)^{l}(1-\rho)^{k-l} \bar{I}_{k-l} \\
& =\sum_{l=1}^{k} C_{k-1}^{l-1}(\rho)^{l-1}(1-\rho)^{k-l} \bar{I}_{k-l}-\sum_{l=1}^{k} C_{k}^{l}(\rho)^{l}(1-\rho)^{k-l} \bar{I}_{k-l}-(1-\rho)^{k}\left(\overline{\mathbb{Y}}_{k}\right)
\end{aligned}
$$

Consider

$$
\begin{aligned}
\frac{\partial F_{k+1}}{\partial \rho}(\rho) & =\sum_{l=0}^{k} C_{k}^{l}\left[l(\rho)^{l-1}(1-\rho)^{k-l}-(k-l)(\rho)^{l}(1-\rho)^{k-l-1}\right] \bar{I}_{k-l} \\
& =\sum_{l=0}^{k} C_{k}^{l}(\rho)^{l-1}(1-\rho)^{k-l-1}(l-k \rho) \bar{I}_{k-l} \\
& =\sum_{l=1}^{k} C_{k}^{l}(\rho)^{l-1}(1-\rho)^{k-l-1}(l-k \rho) \bar{I}_{k-l}-k(1-\rho)^{k-1} \bar{I}_{k},(11)
\end{aligned}
$$

so that

$$
\frac{\partial F_{k+1}}{\partial \rho}(\rho)=\sum_{l=1}^{k} l C_{k}^{l}(\rho)^{l-1}(1-\rho)^{k-l-1} \bar{I}_{k-l}-k \sum_{l=1}^{k} C_{k}^{l}(\rho)^{l}(1-\rho)^{k-l-1} \bar{I}_{k-l}-k(1-\rho)^{k-1} \bar{I}_{k} .
$$

Given that $C_{k-1}^{l-1}=l C_{k}^{l} / k$, using (10) yields:

$$
\frac{\partial F_{k+1}}{\partial \rho}(\rho)=\left(\frac{k}{1-\rho}\right)\left(F_{k}(\rho)-F_{k+1}(\rho)\right)
$$

as claimed.

Properties $\mathbf{2}$ and $\mathbf{3}$ We have that

$$
\begin{aligned}
\frac{\partial F_{k}}{\partial \rho}(\rho) & =\sum_{l=0}^{k-1} C_{k-1}^{l}\left[l(\rho)^{l-1}(1-\rho)^{k-1-l}-(k-1-l)(\rho)^{l}(1-\rho)^{k-l-2}\right] \bar{I}_{k-1-l} \\
& =\sum_{l=1}^{k-1} C_{k-1}^{l}(\rho)^{l-1}(1-\rho)^{k-l-2}[l-(k-1) \rho] \bar{I}_{k-1-l}-(k-1)(1-\rho)^{k-2} \bar{I}_{k-1},
\end{aligned}
$$


So

$$
\frac{\partial F_{k}}{\partial \rho}(0)=-(k-1)\left(\bar{I}_{k-1}-\bar{I}_{k-2}\right)
$$

for $k \geq J+1$.

Denote now $\widehat{I}_{k}(\rho)$ for the expected payoff to an insider when there are $k$ outsiders who choose to enter with probability $\rho$ (the expectation being conditional upon at least one outsider entering). Then

$$
\widehat{I}_{k}(\rho)=\sum_{l=1}^{k} C_{k}^{l} \frac{(\rho)^{l}(1-\rho)^{k-l}}{1-(1-\rho)^{k}} \bar{I}_{k-l}
$$

so straightforward manipulations yield:

$$
\begin{aligned}
\left(1-(1-\rho)^{k}\right) \widehat{I}_{k}(\rho) & =\sum_{l=1}^{k} C_{k}^{l}(\rho)^{l}(1-\rho)^{k-l} \bar{I}_{k-l} \\
& =\sum_{l=0}^{k} C_{k}^{l}(\rho)^{l}(1-\rho)^{k-l} \bar{I}_{k-l}-(1-\rho)^{k} \bar{I}_{k} \\
& =F_{k+1}(\rho)-(1-\rho)^{k} \bar{I}_{k} .
\end{aligned}
$$

If there existed a unique $\rho_{k}$ satisfying $F_{k}\left(\rho_{k}\right)=c_{p}$, then we would have $\widehat{I}_{k}\left(\rho_{k}\right)=$ $\bar{I}_{k}$, so using the previous equality for $\rho=\rho_{k}$ would yield

$$
\left(1-\left(1-\rho_{k}\right)^{k}\right) \bar{I}_{k}=F_{k+1}\left(\rho_{k}\right)-\left(1-\rho_{k}\right)^{k} \bar{I}_{k},
$$

that is, an insider's expected payoff (net of $\Pi_{n}$ ) when $k$ outsiders remain to enter would satisfy

$$
\bar{I}_{k}=F_{k+1}\left(\rho_{k}\right)
$$

if a unique $\rho_{k}$ satisfying $F_{k}\left(\rho_{k}\right)=c_{p}$ existed.

Because we know that there exists a unique $\rho_{J^{\prime}}$ satisfying $F_{J^{\prime}}\left(\rho_{J^{\prime}}\right)=c_{p}$, it simply remains to prove that $\frac{\partial F_{k}}{\partial \rho}(0)>0$, that is, $\bar{I}_{k-1}<\bar{I}_{k-2}$ for $k \geq J^{\prime}+1$, which follows from working recursively on $k$ as in Vettas (2000). ${ }^{13}$

${ }^{13}$ Notice that expressions (4a), (5a) and (6)-(9) in Vettas (2000) are equivalent to expressions (8), (15), (9) for $\rho=0,(12), F_{k}(1)=0<c_{p}$, and (13), respectively. Note 
A direct application of Lemmas A-D yields the following result.

\section{Proposition $1 \mathrm{~b}$}

In the continuous-time limit of the game, the outcome of any symmetric equilibrium exhibits the following properties when $\Pi_{n}>c_{e}$ : there exists a critical number $J^{\prime} \in\{3, \ldots, n\}$ such that, if the gate-keeper initially paid for protection (paid $c_{p}$ and continuously paid $f$ as long as no entry takes place):

1. $J^{\prime} \neq n$ implies that outsiders randomize in such a way that at least $n-J^{\prime}$ of them immediately enter and pay the protection cost $c_{p}$, whereas $J^{\prime}=n$ implies that no outsider enters immediately.

2. After entry by at least $n-J^{\prime}$ outsiders (if $J^{\prime} \neq n$ ) or none of them (if $\left.J^{\prime}=n\right)$, the remaining outsiders, if there are at least two, delay entry for a random length of time and do not pay for protection upon entry.

3. After one of them enters without paying the protection cost, all the remaining outsiders immediately follow at no cost.

This proposition also holds when $\Pi_{n} \leq c_{e}$, a situation that for large enough values of $n$ approximates free entry. In particular, there exists a critical value $\bar{J}$ such that if the number of outsiders is larger or equal to $\bar{J}$, outsiders mix between actions $p$ and $w$ and at least $n-\bar{J}$ immediately enter if $\bar{J} \neq n$ or none enter if $\bar{J}=n$. The main difference is that if the number of outsiders is less than $\bar{J}$, no further entry takes place whereas in the case $c_{e}<\Pi_{n}$, all players play a waiting game and eventually enter. We derive the value of $\bar{J}$ below.

In situations where $c_{e} \geq \Pi_{n}$, action $u$ is always dominated by $w$ if no insider has ever stopped paying for protection (both upon entry and thereafter), since playing $u$ yields payoff $\Pi_{n}-c_{e} \leq 0$. Define $c_{k}^{\prime} \equiv \Pi_{n-k}-c_{e}$ in what follows, and let $\bar{J} \equiv \max \left(J_{1}, J_{2}^{\prime}\right)$, where $J_{2}^{\prime} \equiv \inf \left\{k \geq 3: c_{p}<c_{k-1}^{\prime}\right\}$ (with $J_{2}^{\prime}=n$ if the definition is vacuous), so that subgames with $k \leq \bar{J}-1$ outsiders exhibit

that the expression that turns out to be equivalent in our setting to (10) in Vettas (2000) (namely, $F_{k+1}(0)>\bar{I}_{k}$ ) actually holds with equality, and hence it is redundant based on the expression in (9) evaluated at $\rho=0$. 
no further entry. ${ }^{14}$ Lemmas A-D are then directly applicable by simply letting $c_{e}=\Pi_{n}$ and redefining $c_{k, F}^{*}$ and $J_{2}$ as $c_{k}^{\prime} \equiv \Pi_{n-k}-c_{e}$ and $J_{2}^{\prime}$, respectively. Hence, the case in which $c_{e} \geq \Pi_{n}$ corresponds to infinitely long entry delays in subgames in which insiders continuously pay $f$ and outsiders have no preemptive incentives. The proposition then applies accounting for this new notation and the fact that the delay in entry after the initial preemptive entry phase is infinite.

\footnotetext{
${ }^{14}$ Since it holds that $\Pi_{n-(\bar{J}-1)}-c_{e}>c_{p} \geq \Pi_{n-(\bar{J}-2)}-c_{e}$ by definition of $\bar{J}$.
} 